\title{
Frequency-Domain Measurement of the Millimeter Wave Indoor Radio Channel
}

\author{
Peter F. M. Smulders, Member, IEEE, and Anthony G. Wagemans
}

\begin{abstract}
Coherent wideband frequency-domain measurements of the complex frequency response of millimeter wave indoor radio channels are discussed. In addition, results of measurements performed in a $2 \mathrm{GHz}$ band centered around 58 $\mathrm{GHz}$ will be presented. It is shown that a $40 \mathrm{~dB}$ dynamic range and a $400 \mathrm{~ns}$ aliasing-free range are sufficient for a correct estimation of the rms delay spread from the measurement data.
\end{abstract}

\section{INTRODUCTION}

$\mathbf{R}$ ECENTLY, THERE has been significant interest in indoor wireless communications using the millimeter wave frequency bands lying around $60 \mathrm{GHz}$. The key attractions of these bands are the potentially vast communication capacity and the promise of short frequency reuse distances. In order to attain insight in the propagation characteristics of millimeter wave indoor radio channels, coherent wideband frequencydomain measurements were carried out in various indoor areas at the Eindhoven University of Technology [1].

Frequency-domain sounding is a coherent measurement technique that can be performed with 1) a frequency-sweep stimulus or 2) a frequency-stepping stimulus. In the frequencysweep technique the source is swept in a continuous fashion from the lower to the upper frequency, and the received signal is sampled without stopping the sweep. In the frequencystepping technique a single sine wave originating from a synthesized source is transmitted, and the received signal amplitude and phase are detected at the receiving end. Repeating this procedure by stepping through the frequency band with constant transmitted power produces the equivalent low-pass transfer function of the channel. Applying Inverse Fourier Transformation (IFT) yields the corresponding equivalent low-pass impulse response. The frequency-sweep method provides fast measurements in comparison with the frequencystepping method. The frequency-stepping method, however, provides the benefit of improved frequency accuracy since the source is tuned and phase-locked to each frequency point. With both frequency-domain techniques, the achievable timedomain resolution depends only on the channel bandwidth and the frequency-domain window applied to improve the dynamic range in the time domain. A drawback of the frequencystepping method is, in general, that the channel must be fixed during a complete measurement sweep in order to obtain meaningful results. As a consequence, no Doppler effects can

Manuscript received September 18, 1992; revised July 20, 1994. This work was supported in part by STW (Stichting Technische Wetenschappen).

The authors are with the Department of Electrical Engineering, Eindhoven University of Technology, Room EH 11.02, NL 5600 MB Eindhoven, The Netherlands.

IEEE Log Number 9414835.

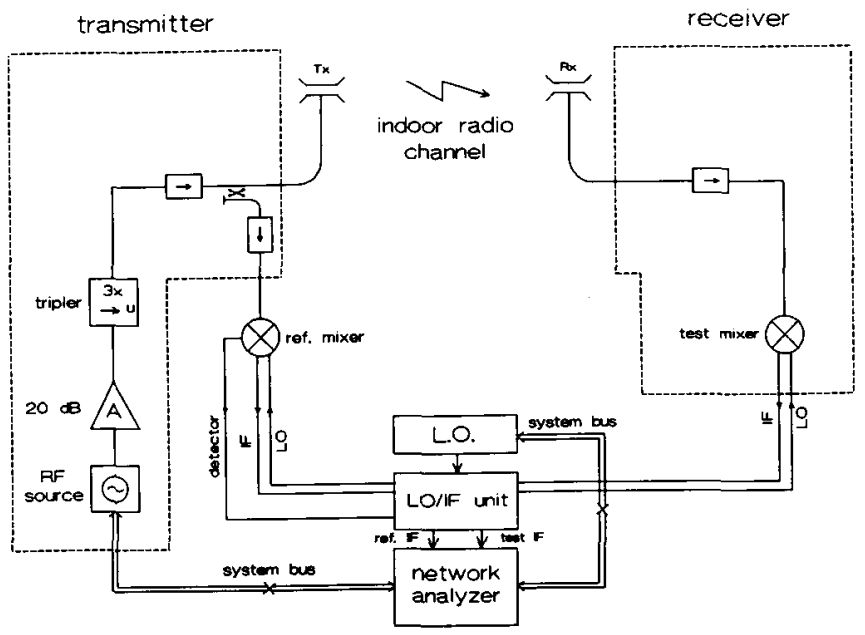

Fig. 1. Measurement setup.

be measured. We, however, applied the frequency-stepping method because of the high measurement accuracy obtained.

In Section II, the measurement equipment used for conducting the indoor propagation experiments will be described. In Section III, the type of antennas used for experiments is discussed briefly. In Section IV, the channel parameters considered are defined. In Section V, three of the rooms in which measurements were performed will be described, and measurement results will be presented and discussed.

\section{Measurement SETUP}

Our system for conducting wideband indoor radio propagation measurements is built up around the HP 8510C network analyzer. A schematic diagram of the setup is drawn in Fig. 1.

The network analyzer controls the RF source by an IEEE bus. The RF source is capable of generating sine waves only up to a frequency of $50 \mathrm{GHz}$. To increase the frequency range of the setup, the output frequency of the RF source is tripled. A $20 \mathrm{~dB}$ amplifier must be inserted between the RF source and the tripler to obtain the power level required for proper operation of the tripler. The output of the tripler is applied to a $-10 \mathrm{~dB}$ directional coupler. The directional coupler separates the incoming signal into the reference signal and the test signal which run via the reference channel and test channel, respectively. The reference channel provides the analyzer with a phase reference. The reference output of the directional coupler is connected via an isolator and a rectangular WR19 waveguide to the harmonic mixer. The test output of the directional coupler is connected to the transmit antenna. The 
receive antenna is connected to the harmonic mixer of the test channel via an isolator. Both antennas are biconical horn antennas which exhibit an omnidirectional radiation pattern in the horizontal plane [2].

The local oscillator/intermediate frequency (LO/IF) unit uses a power splitter to feed one local oscillator signal, which ranges from 4 to $6 \mathrm{GHz}$, to the two harmonic mixers. Flexible coaxial cables connect the mixers to the LO/IF unit. This allows flexible displacement of the transmitter and receiver. The network analyzer system is extended by the lengths of the cables, i.e. the separation distance between the test ports is increased. The unit controls the level of LO power at the input of the reference mixer. This is possible by means of a feedback circuit which includes a detector at the mixer LO input. The harmonic mixers multiply the 10th harmonic of the LO signal with the RF frequency. The LO frequency is offset by $20 \mathrm{MHz}$; hence a $20 \mathrm{MHz}$ IF signal is obtained from both reference and test channel. Both IF signals are fed back to the network analyzer, where the complex ratio of the signals is calculated and displayed as the $S_{21}$ two-port transmission parameter.

The receiver bandwidth is $10 \mathrm{kHz}$, which results in a noise floor of approximately $-100 \mathrm{dBm}$. At every measurement frequency the receiver noise floor was reduced even further by averaging the response 128 times. This resulted in a noise floor of $-120 \mathrm{dBm}$, thus an increase of the dynamic range by $20 \mathrm{~dB}$. The average level of received power was approximately $-80 \mathrm{dBm}$ for all measurements. Thus a $40 \mathrm{~dB}$ signal-tonoise ratio was attained. This means that multipath fades to a minimum level of $-40 \mathrm{~dB}$ could be detected. The specification of the (linear) magnitude uncertainty, defined as the maximum relative error in the magnitude of the frequency response due to IF residuals, detector inaccuracies, mixer noise figure and conversion loss and due to the effects of cable instability, was approximately 0.02 . This limits the difference between maximum and minimum ray amplitude to $-20 \log (0.02)=$ $34 \mathrm{~dB}$. In many impulse responses, however, differences in ray amplitude of about $40 \mathrm{~dB}$ could be observed.

Before any measurement can be done, calibration of the measuring equipment is necessary. A calibration removes systematic errors in the setup by a reference measurement. There is an essential difference in calibrating the system for measuring antenna radiation patterns as compared to indoor propagation experiments. In the first case, both antennas are connected and placed in an anechoic chamber. The $S_{21}$ response is measured as a reference, and all deviations from an ideal $0 \mathrm{~dB}$ magnitude and 0 phase frequency response are used for mathematically correcting subsequent measurements of $S_{21}$. This is done by multiplying the new $S_{21}$ response with the inverse of the complex reference $S_{21}$ at each measurement frequency. The deviations could be caused by residual reflections against walls and objects in the room or by delays and reflections in cables and waveguides.

This method of calibration is not suited for indoor propagation measurements, because all relevant information on wall and object reflection will be lost. Instead of this, the calibration is performed by removing both antennas and connecting a piece of waveguide between the two "test ports," i.e., coupler output and test channel mixer. A "through" response measurement is then used as a reference. In doing so, systematic errors caused by the equipment are mathematically removed. The "through" response appears as a unit delta pulse at $\tau=0$ ns excess delay. The moment $\tau=0$ can therefore be considered as a common origin for all subsequent time-domain measurement results. The significance of this is that measured impulse responses do not have the line-of-sight (LOS) ray displayed at $\tau=0$ seconds; hence profiles have to be shifted in time on the analyzer display before they are stored. If the LOS ray can't be clearly discerned in a measured response, its position can be checked by placing a piece of absorber in the LOS path and noting the response change.

The network analyzer is capable of taking a maximum of 801 frequency samples. This number and the measurement bandwidth determine the aliasing-free range in the time domain:

$$
\text { Range }=\frac{N-1}{B}
$$

where $N$ is the number of frequency samples, and $B$ is the measurement bandwidth. In order to investigate the worstcase (maximum) impulse response excess delay we performed preliminary experiments in various indoor areas which indicated that $400 \mathrm{~ns}$ should be sufficient for measuring impulse responses. According to (1) the maximum bandwidth necessary for a $400 \mathrm{~ns}$ aliasing-free range with full accuracy is therefore $2 \mathrm{GHz}$. The Discrete Fourier Transform of the equally spaced frequency samples yields 801 complex timedomain data points which are also equally spaced at $1 / B=$ $0.5 \mathrm{~ns}$ intervals.

Frequency data can be shaped (multiplied with a function) in order to improve the dynamic range in the time domain. Because the measurements are performed within a specified bandwidth, no shaping is a rectangular frequency-domain window by definition. It is well known that multiplying frequency data with a window function is a convolution with the IFT of the window function in the time domain. For example, if no additional windowing is used, each timedomain pulse is a sinc function $B(\sin (\pi B t)) /(\pi B t)$. Because the maximum side-lobe level of a sinc is $13 \mathrm{~dB}$ below the main-lobe level, the time-domain dynamic range will be limited to $13 \mathrm{~dB}$ in this case. A rectangular window offers, however, the highest time-domain resolution. Time-domain resolution can be defined as the minimum time distance at which two pulses of equal amplitude are still discernible. It is generally equated with the width of the main lobe of the IFT of the window function. With a rectangular window and $B=$ $2 \mathrm{GHz}$ the time-domain resolution is $1 / B=0.5 \mathrm{~ns}$.

Generally, dynamic range and resolution are two opposing requirements. Using a window, therefore, means finding a compromise between the required resolution and dynamic range. The network analyzer uses a Kaiser window [3] for shaping frequency samples. This window has a highest sidelobe level of $-44 \mathrm{~dB}$, and its main lobe is slightly broader than that of a rectangular window. Using a Kaiser window and a measurement bandwidth of $2 \mathrm{GHz}$ yields a time-domain resolution of $1 \mathrm{~ns}$. 


\section{ANTENNAS}

Considerable attention has been paid to the design of millimeter wave antennas for our measurement setup. The antennas can be considered as a part of the radio channel under consideration, and hence their radiation patterns will have great impact on the channel's propagation characteristics. Therefore, propagation measurement results cannot be well interpreted without a good description of the antenna radiation patterns.

For our measurements we designed a pair of biconical horn antennas which exhibit an omnidirectional radiation pattern in the azimuth (horizontal) plane and a narrow beam in the elevation (vertical) plane. Both biconical horns have a $3 \mathrm{~dB}$ beamwidth of $9^{\circ}$ in the elevation plane and a $9 \mathrm{~dB}$ directivity. The average standing-wave ratio was better than 1.4 in the 2 $\mathrm{GHz}$ bandwidth. These antennas are, in fact, scaled versions of the ones treated in [2].

The main reason for using antennas that radiate omnidirectional in azimuth is that a base station antenna located in the center of an office near the ceiling should give the same coverage in every direction in the room, since this contributes to fair access to every user in a room. An omnidirectional antenna on a remote terminal offers the advantage of avoiding cumbersome pointing of the remote antenna towards the base station antenna. The difference in height of the base station antenna and the remote terminal antenna in combination with the directivity in elevation might result in a more or less uniform distribution for the received power throughout the coverage area due to antenna gain compensation of path losses [2].

A drawback of the application of biconical horn antennas, when compared with the application of non-omnidirectional high-gain antennas, is the expected stronger multipath component. This component causes severe intersymbol interference which limits the maximum achievable bit rate. Therefore, it makes sense to perform additional experiments with high-gain antennas. Such experiments are reported in [4].

\section{INDOOR RADIO CHANNEL DEFINITION}

It has been shown [5]-[8] that an indoor radio channel operating in the UHF-band is essentially a multipath channel. This means that a single transmitted pulse will be received as a number of delayed and attenuated pulses. These pulses are caused by rays following discrete paths. This ray occurrence process is likely to be even more pronounced for the much shorter millimeter waves. This is confirmed by our measurement results in which individual rays could be resolved due to a measurement resolution of $1 \mathrm{~ns}$.

The complex equivalent low-pass impulse response of a millimeter wave indoor radio channel can therefore be considered as discrete, so that it may be written as

$$
h(\tau)=\sum_{n} \beta_{n} e^{j \theta_{n}} \delta\left(\tau-\tau_{n}\right)
$$

where $\beta_{n}, \theta_{n}$, and $\tau_{n}$ are the amplitude (normalized on the amplitude of the transmitted pulse), the phase and the excess delay of ray $n$, respectively. These parameters will be timevariant, due to movements in the room. However, since motion speed is expected to be low, the channel response will not noticeably change over many bit periods. The channel is therefore assumed to be quasi-static.

The power delay profile is defined as [5]

$$
p(\tau) \triangle|h(\tau)|^{2} .
$$

If pulses do not overlap at the receiver, then we may write

$$
p(\tau)=\sum_{n} \beta_{n}^{2} b^{2}\left(\tau-\tau_{n}\right)
$$

In (4) $b(\tau)$ is the base-band pulse shape, which is in our case the Inverse Fourier Transform of the applied frequencydomain window function. If the ray phases $\theta_{n}$ are assumed to be uniformly distributed over $[0,2 \pi]$, then the mathematical expectation of $p(\tau)$ yields the same result [7]. This assumption was checked for all measurement series and found to be true.

A measure for the temporal channel dispersion is the rms delay spread defined as [5]

$$
\sigma \triangle \sqrt{\left(\overline{\tau^{2}}\right)-(\bar{\tau})^{2}}
$$

where

$$
\overline{\tau^{p}}=\frac{\sum_{n} \beta_{n}^{2} \tau_{n}^{p}}{\sum_{n} \beta_{n}^{2}}, \quad p=1,2 .
$$

The rms delay spread is a rough measure for the feasible bit rate in the case no sophisticated techniques such as channel equalization are applied.

The denominator of (6) is the normalized received power which is defined as the ratio of the total received power $P_{r}$ and the transmitted power $P_{t}$, hence

$$
\frac{P_{r}}{P_{t}} \triangle \sum_{n} \beta_{n}^{2}
$$

The normalized received power can be used for estimating the signal-to-noise ratio in an indoor radio communication system. It is also important for determining the radio coverage in a particular room.

An average of all measured profiles in a particular room can be defined as

$$
P_{\mathrm{av}}(\tau) \unlhd \frac{1}{K} \sum_{k=1}^{K} \frac{p_{k}(\tau)}{\sum_{n} \beta_{k, n}^{2}}
$$

in which $K$ denotes the total number of profiles obtained in the room considered, whereas $p_{k}(\tau)$ represents the $k$ th individual power profile with $k=1,2, \cdots K . \beta_{k, n}$ represents the $n$th ray of the $k$ th individual impulse response. In calculating the average power delay profile, each individual profile is thus weighted by its own normalized received power. This is done because a single profile should not dominate the average delay profile. 


\section{Measurement Results}

We will consider results of measurements carried out in three different indoor areas at the Eindhoven University of Technology. These areas consist of one single room only, because millimeter waves are severely attenuated by most inner walls. In the following these rooms are denoted $F, G$ and $\mathrm{H}$.

Room F ("Computer room" in the Computer Center Building) is a rectangular room with dimensions $9.9 \times 8.7 \times 3.1 \mathrm{~m}^{3}$ One of he long sides consists of large floor-to-ceiling doubleglazed windows encased in a steel frame, while the other sides are smooth metal walls. Some 20 personal computers are lined up against these walls. Wooden chairs and tables with metal legs are lined up in the room. The measured "return loss coefficient" (magnitude) of the most dominant wall material (metal) is $R=0 \mathrm{~dB}$.

Room G ("Lecture room" in the Auditorium Building) is a typical rectangular classroom with dimensions $12.9 \times 8.9$ $\times 4.0 \mathrm{~m}^{3}$. One of the long sides consists of large windows in a steel framework from $1 \mathrm{~m}$ height to ceiling. Up to $1 \mathrm{~m}$ height there is concrete. The other sides are walls which are completely covered with wooden lathing. The concrete floor is covered with linoleum. The ceiling is covered with lighting holders. Tables and chairs are lined up throughout the room. The measured return loss coefficient of the most dominant wall material (wooden panels) is about $R=8 \mathrm{~dB}$.

Room H ("Laboratory room" in the Electrical Faculty Building) is rectangular with dimensions $11.3 \times 7.3 \times 3.1$ $\mathrm{m}^{3}$. One of the short walls consists of a window from $1 \mathrm{~m}$ height to ceiling and a metal heating radiator case below. The other walls consist of smoothly plastered concrete. The floor is linoleum on concrete. Four square $\left(0.5 \times 0.5 \mathrm{~m}^{2}\right)$ concrete pillars and a metal cupboard in a corner are dominant objects in this room. A number of large wooden benches with wooden shelves on top are located in this room. The ceiling consists of aluminum plates and lighting holders. The measured return loss coefficient of the most dominant wall material (concrete) is $R=2 \mathrm{~dB}$.

In each room at least one series of 20 measurements at $57-59 \mathrm{GHz}$ was conducted at randomly chosen locations of the transmitter (being a trolley on which the transmitting antenna was fixed) in the room. At every measurement position a lineof-sight connection between the transmit and receive antenna was present. The biconical horn antenna at the transmitting end was located at $1.4 \mathrm{~m}$ above the floor. The biconical horn at the receiving end was elevated to $3 \mathrm{~m}$. The base station was placed in the center of the room. An example of a frequency response for a single measurement position in Room $G$ is presented in Fig. 2. The corresponding power delay profile is shown in Fig. 3.

In Fig. 4 the measured average power delay profiles in Environments F, G, and $\mathrm{H}$ are depicted. For large excess delay ( $\tau>350 \mathrm{~ns}$ ) all profiles converge to the system noise floor. For $\tau>50 \mathrm{~ns}$ the received power in $\mathrm{dB}$ decays linearly. This linear decaying part is not only observed in the average power delay profile, but also in most individual power delay profiles. The difference in slopes can be attributed to differences in

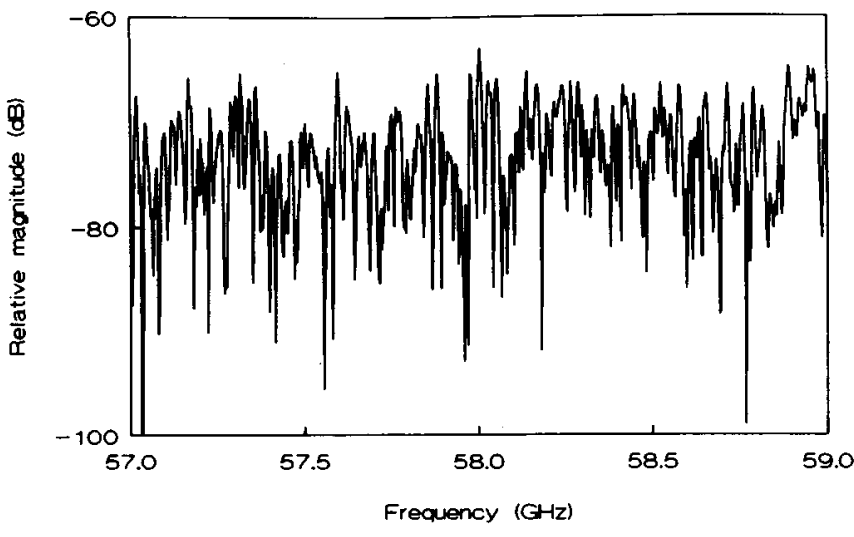

Fig. 2. Typical measured $S_{21}$ response versus frequency in Room G.

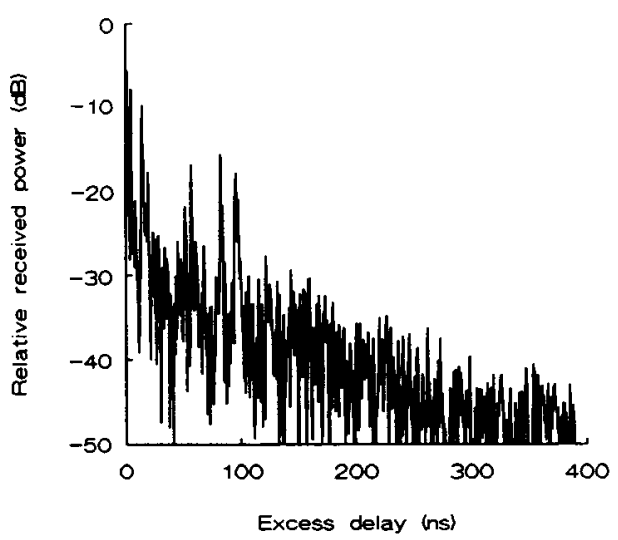

Fig. 3. Measured power delay profile.

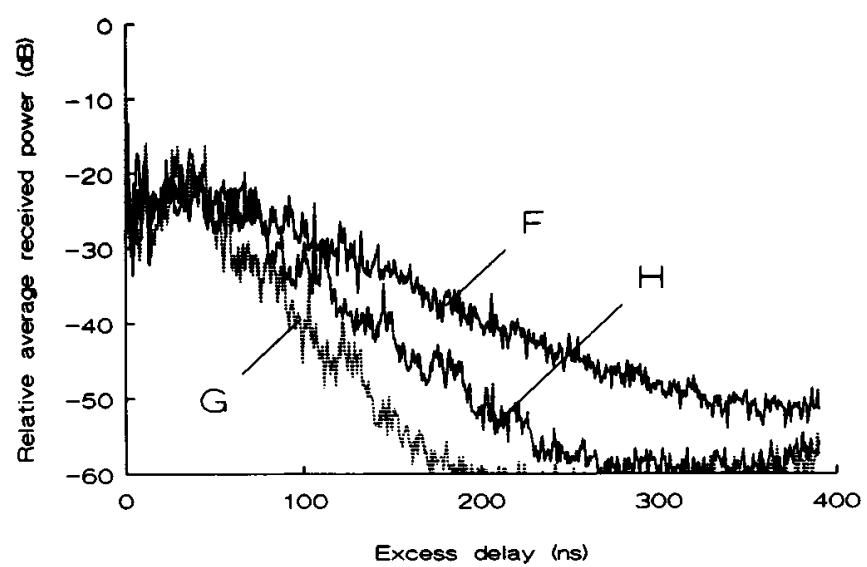

Fig. 4. Measured average power delay profiles in Room F, G, and $H$

reflection coefficients of dominant wall partitions. For small excess delay ( $\tau<60 \mathrm{~ns}$ ) the power in the average profiles does not follow the same linear decay, but remains more or less constant. This is caused by antenna gain compensation in elevation of the free-space losses as explained in [2].

In Fig. 5 the (complementary) cumulative distribution functions for the rms delay spread values obtained in Room $F, G$, and $\mathrm{H}$ are shown. In Section II it was shown that with 801 data samples in a $2 \mathrm{GHz}$ bandwidth an aliasing-free range of exactly $400 \mathrm{~ns}$ is achieved. Because of the convolution with 


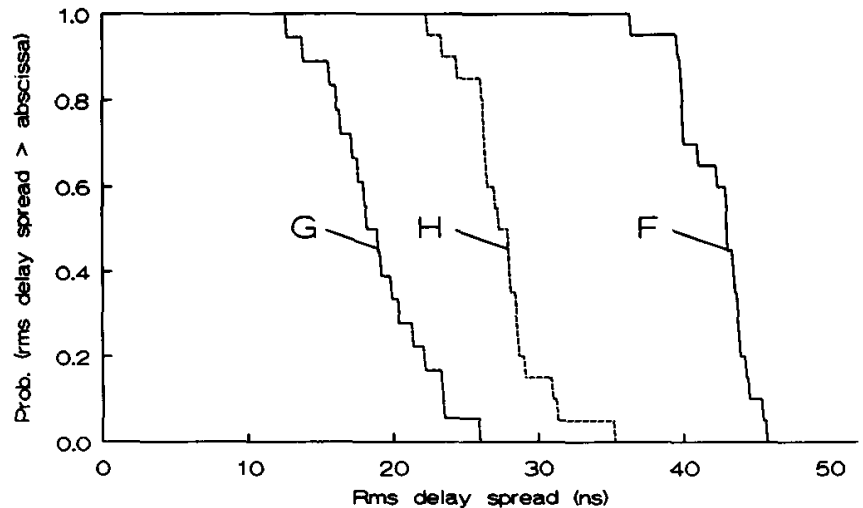

Fig. 5. Rms delay spread statistics for Room F, G, and H.

a window function, each pulse in the profile consists of more than one data point. Because the maximum of the line-of-sight pulse is located at $\tau=0 \mathrm{~ns}$, a few data points of this first ray will have negative time values. Due to aliasing, these values fold back to values at $400 \mathrm{~ns}$. Although these data points do contribute to the total signal power, they should not be used for calculation of the rms delay spread, because for this channel parameter each ray is weighed with its excess delay. To ensure that the rms delay spread values are not calculated incorrectly, only time values up to $390 \mathrm{~ns}$ are considered.

The rms delay spread statistics presented in Fig. 5 have been calculated using all data points from 0 to 390 ns. Values of 13-45 ns have been found in these rooms. By comparing the curves $\mathrm{F}, \mathrm{G}$, and $\mathrm{H}$ in Fig. 5, it is clear that the rms delay spread increases with the reflectivity of the walls. Fig. 5 indicates that the rms delay spread values remain fairly constant throughout a particular room. For characterization of rms delay spread values in a room by a single delay spread value, an average rms delay spread is defined. This average spread is calculated using the average power delay profile defined in Section IV. The average rms delay spread is not exactly equal to, but very similar to the average of the individual rms delay spread values.

The influence of noise, residual channel dispersion and profile truncation at high excess delays on rms delay spread calculation can be evaluated by reducing the maximum excess delay time up to which data points are included in the calculation of (5) and (6). In Fig. 6 the average rms delay spread as a function of maximum excess delay is drawn for the measurement series in Room F, G, and H at 57-59 GHz. From Fig. 6 it is clear that the average rms delay spread does not increase very much once the average power delay profile has dropped by $40 \mathrm{~dB}$. This implies that a $40 \mathrm{~dB}$ dynamic range and a 400 ns aliasing-free range are sufficient for correct determination of the rms delay spread in the considered indoor areas.

\section{SUMmaRY AND CONCLUSIONS}

Wideband measurements of millimeter wave indoor radio channels based on a frequency-stepping technique are discussed. The heart of the measurement system is a network analyzer which is capable of measuring complex values of two-port $S$ parameters. From these, channel characteristics such as rms delay spread and (normalized) received power

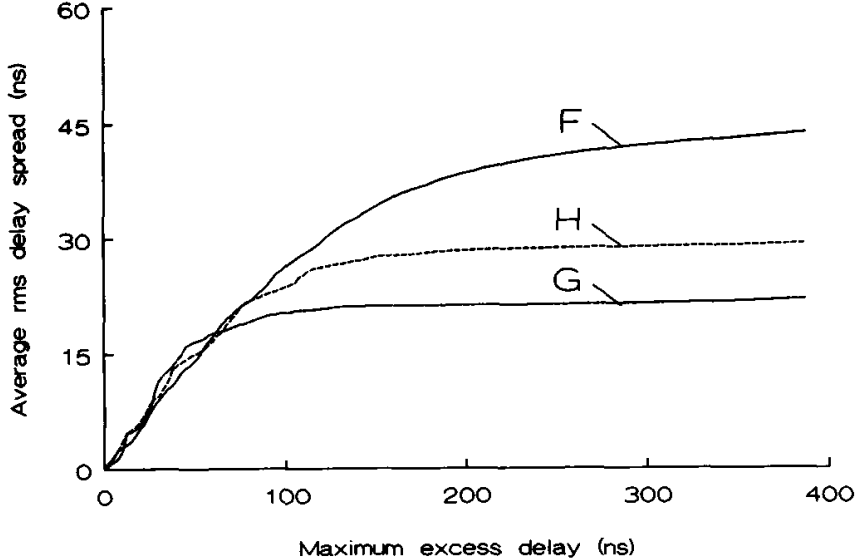

Fig. 6. Increase of average power delay profile with maximum excess delay in Room $\mathrm{F}, \mathrm{G}$, and $\mathrm{H}$.

can be derived. One of the most critical issues which might influence the measurement results would be the antennas, because their radiation patterns will have great impact on the indoor radio channel characteristics, such as delay spread and received power. In our setup biconical horn antennas were used. These antennas exhibit an omnidirectional radiation pattern in the azimuth plane.

Measurement results of experiments in three different rooms at the Eindhoven University of Technology, in a $2 \mathrm{GHz}$ bandwidth centered around $58 \mathrm{GHz}$, have been presented. With respect to these multipath radio channels, it is shown that a $40 \mathrm{~dB}$ dynamic range and a $400 \mathrm{~ns}$ aliasing-free range are sufficient for a correct derivation of the rms delay spread from the measurement data obtained.

\section{ACKNOWLEDGMENT}

The authors wish to thank Prof. Dr. G. Brussaard and Dr. M. Herben for their helpful suggestions in preparing this article. We also wish to thank STW (Stichting Technische Wetenschappen) for their financial support which made it possible for the Telecommunications Division of the Eindhoven University of Technology to purchase the expensive measurement equipment.

\section{REFERENCES}

[1] P. F. M. Smulders and A. G. Wagemans, "Wideband indoor radio propagation measurements at $58 \mathrm{GHz}$," Electron. Lett., vol. 28, no. 13, pp. 1270-1271, June 1992 .

[2] "Biconical horn antennas for uniform coverage in indoor areas at mm-wave frequencies," IEEE Trans. Veh. Technol, vol. 43, no. 4, pp. 897-901, Nov. 1994.

[3] F. F. Kuo and J. F. Kaiser, System Analysis by Digital Computer. New York: Wiley, 1966.

[4] P. F. M. Smulders and A. G. Wagemans, "Wide-band measurements of $58 \mathrm{GHz}$ indoor radio channels," in URSI, Proc. 3th Int. Symp. on Signals, Systems and Electronics, Sept. 1992, pp. 692-696.

[5] A. A. M. Saleh and R. A. Valenzuela, "A statistical model for indoor multipath propagation," IEEE J. Selected Areas Commun., vol. SAC-5, no. 2, pp. 128-137, Feb. 1987.

[6] D. M. J. Devasirvatham "Time delay spread and signal level measurements of $850 \mathrm{MHz}$ radio waves in building environments," IEEE Trans. Antenn. Propagat., vol. AP-34, pp. 1300-1308, Nov. 1986.

[7] P. Yegani and C. D. McGillem, "A statistical model for the factory radio channel," IEEE Trans. Commun., vol. 39, pp. 1445-1454, Oct. 1991.

[8] T. S. Rappaport and C. D. McGillem, "UHF fading in factories," IEEE J. Selected Areas Commun., vol. 7, no. 1, pp. 40-48, Jan. 1989. 


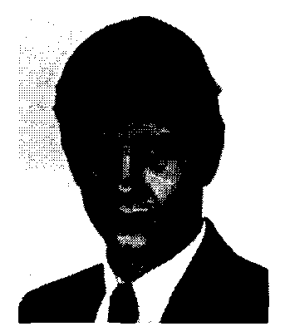

Peter F. M. Smulders (S' $82-$ M-92) was born in Eindhoven, The Netherlands, on May 23, 1957. He graduated from Eindhoven University of Technology in 1985 .

In September 1985 he joined the Propagation and Electromagnetic Compatibility Department of the Research Neher Laboratories of the Netherlands PTT. While he was with PTT he was doing research in the field of compromising emanation from civil data processing equipment. Since June 1988 he has been with the Telecommunication Division of Eindhoven University of Technology. His current interests deal with digital satellite and land mobile communications. In addition, he pursued the Ph.D. degree in the field of broadband in Wireless LAN's. He has several publications in this area. He is currently involved in the ACTS project MEDIAN-Wireless Broadband CPN/WLAN for Professional and Residential Multimedia Applications.

Mr. Smulders is a member of the working group of the European cooperation in the field of scientific and technical research (COST 231) on a project dealing with "Evolution of the land mobile radio (including personal) Communications." He is co-founder and present board member of the James Clerk Maxwell Foundation. He is a member of the Executive Committee of the IEEE Vehicular Technology/Communications Society Joint Chapter, Benelux Section.

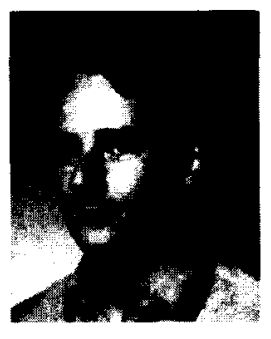

Anthony G. Wagemans was born in Sittard, The Netherlands, on April 8, 1966. At the age of ten he emigrated to Cape Town, South Africa, where he stayed for five years. In February 1992 he graduated cum laude at the Telecommunications Division of The Eindhoven University of Technology.

Mr. Wagemans has fulfilled his military service as a reserve officer of the Royal Dutch Navy, tutoring electronics at the Royal Netherlands Naval College in Den Helder. Since September 1993 he has been with Philips Research. 